\title{
RETIFICADOR PRÉ-REGULADOR BOOST COM ELEVADOS FATOR DE POTÊNCIA E RENDIMENTO, PARA SISTEMAS DE TELECOMUNICAÇÕES
}

\author{
Fabio Toshiaki Wakabayashi \\ Universidade Estadual Paulista \\ UNESP - FEIS - DEE \\ Cx. Postal 31 - 15385-000 - Ilha Solteira (SP) \\ Fax: (18) 37422735 \\ e-mail: canesin@dee.feis.unesp.br \\ http://www.dee.feis.unesp.br/lep/power.html
}

Carlos Alberto Canesin

\begin{abstract}
Resumo - Este artigo apresenta, de forma resumida, as variações topológicas de uma célula de comutação ZCSPWM, a partir da análise de suas aplicações em estágios retificadores pré-reguladores Boost controlados pela técnica de valores médios instantâneos de corrente de entrada, com o propósito de obter uma estrutura retificadora com elevados rendimento e fator de potência para alimentação monofásica de sistemas de telecomunicações. As principais características de cada uma das versões da célula de comutação são descritas, com o intuito de propiciar uma comparação qualitativa entre as estruturas analisadas. Adicionalmente, são apresentados resultados experimentais para a mais recente versão do retificador Boost ZCS-PWM, implementado para o processamento de valores nominais de $1200 \mathrm{~W}$ de potência de saída, com $220 \mathrm{~V}$ de tensão eficaz de alimentação, $400 \mathrm{~V}$ de tensão média de saída e $50 \mathrm{kHz}$ de freqüência de chaveamento.
\end{abstract}

\begin{abstract}
This paper presents a summary of different topological arrangements concerned to a ZCS-PWM commutation cell, based on the analysis of its application in Boost rectifying pre-regulators, controlled by the technique of instantaneous average values of input current, with the purpose to obtain a high input powerfactor rectifier, and high efficiency to single-phase application in telecommunication systems. The main characteristics of each commutation cell are described, providing conditions to establish a qualitative comparison among the structures. In addition, experimental results are presented from a prototype of the latest version of the ZCS-PWM Boost rectifier, implemented for processing nominal values of $1200 \mathrm{~W}$ output power and $400 \mathrm{~V}$ average output voltage, at $220 \mathrm{~V}$ rms input voltage and $50 \mathrm{kHz}$ switching frequency.
\end{abstract}

\section{INTRODUÇÃO}

A crescente demanda mundial por energia elétrica tem feito com que questões referentes ao planejamento de sua produção, transmissão e consumo tornem-se mais claras à população, de maneira geral. Dentro deste contexto, a adoção de políticas de racionalização do consumo de energia elétrica tem sido incentivada. Uma das formas de se racionalizar o consumo é obtida a partir do uso de equipamentos eletroeletrônicos com processamento de energia otimizado, ou seja, equipamentos que apresentam elevados rendimento e fator de potência, caracterizando assim um melhor uso da energia elétrica. É fato que melhores rendimentos resultam no aumento da densidade de potência destes equipamentos, possibilitando sua compactação. Em relação ao fator de potência, a obtenção de elevados valores para esta grandeza implica na redução do conteúdo harmônico das correntes drenadas por tais equipamentos da rede de corrente alternada (CA). Além disto, elevar o fator de potência de uma estrutura significa reduzir a diferença entre os valores de potência consumida $(\mathrm{kW})$ e potência demandada da rede de CA (kVA), na busca da igualdade entre tais valores.

Dentre a infinidade de equipamentos eletro-eletrônicos, existem aqueles que necessitam de um estágio de entrada retificador, conectado entre a rede de alimentação em CA e a carga propriamente dita. Considerando sua configuração mais simples, o estágio retificador é geralmente composto por uma ponte de diodos associada a um capacitor de filtro de elevado valor, conforme mostra a figura 1 para uma aplicação em sistemas monofásicos. Tipicamente, equipamentos que utilizam esta configuração apresentam fator de potência bastante reduzido, da ordem de 0,6 . Tal fato denota a má utilização da energia total drenada da rede de alimentação em CA.

Como uma das alternativas para a minimização deste problema, destacam-se os estudos desenvolvidos nos últimos vinte anos de estruturas retificadoras chaveadas em elevada freqüência, baseadas em conversores estáticos controlados através de técnicas especiais [1 até 6], capazes de propiciar a obtenção de reduzida taxa de distorção harmônica (TDH) na corrente de entrada, além de defasagem angular desprezível entre as componentes fundamentais da tensão de alimentação e da corrente drenada da rede em CA, resultando em elevado fator de potência para a estrutura. Adicionalmente, o chaveamento em elevadas freqüências permite a redução do volume e do peso dos elementos reativos empregados, possibilitando o aumento da densidade de potência processada através do estágio retificador.

Em função de restrições impostas por normas internacionais, tais como as atuais IECs 61000-3-2 e 61000-3-4, o conversor Boost é um dos conversores atuais mais utilizados para a implementação de estágios retificadores monofásicos de elevado fator de potência, conforme mostra a figura 2.

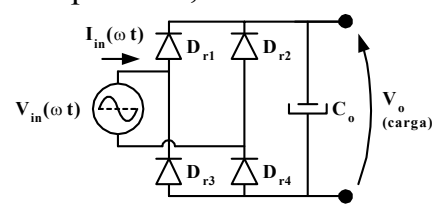

Figura 1 - Estágio retificador convencional, para sistemas monofásicos. 


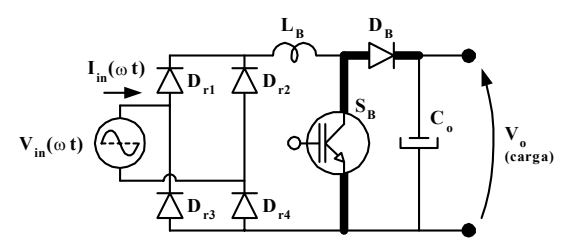

Figura 2 - Estágio retificador Boost de elevado fator de potência, para sistemas monofásicos.

Dentre as características que favorecem a escolha do conversor Boost, destacam-se a simplicidade do circuito e a presença de um filtro de corrente $\left(\mathrm{L}_{\mathrm{in}}\right)$ na entrada da estrutura. Em função disto, dependendo da técnica de controle adotada para o comando do interruptor ativo $S_{B}$, torna-se possível minimizar o filtro de entrada necessário para a eliminação das componentes de elevadas freqüências da corrente drenada da rede de alimentação em CA.

Apesar de proporcionar a redução do volume dos elementos reativos necessários, a operação em elevadas freqüências pode acarretar problemas com relação ao rendimento da estrutura, uma vez que as perdas durante os processos de comutação dos semicondutores envolvidos são diretamente proporcionais à freqüência de chaveamento à qual estão submetidos. Adicionalmente, uma vez que as tensões médias de saída da estrutura retificadora Boost são elevadas (tipicamente $400 \mathrm{~V}$ ), torna-se necessário empregar diodos Boost de elevadas tensões de ruptura e do tipo ultrarápidos (operação em elevadas freqüências), resultando em perdas adicionais e problemas de interferência eletromagnética, devido à recuperação reversa.

Assim sendo, com o intuito de propiciar o aumento da freqüência de operação dos conversores estáticos, sem que haja prejuízo do rendimento dos mesmos, e também em função dos problemas de recuperação reversa dos diodos, técnicas de comutação não-dissipativa têm sido desenvolvidas [7 até 15]. Basicamente, as técnicas de comutação nãodissipativas podem ser divididas em dois grandes grupos:

- técnicas de comutação com tensão nula: ZVS (Zero Voltage Switching) e ZVT (Zero Voltage Transition);

- técnicas de comutação com corrente nula: ZCS (Zero Current Switching) e ZCT (Zero Current Transition).

Cada uma das técnicas de comutação suave é obtida a partir de uma nova configuração de célula de comutação. É fato que a escolha da técnica de comutação a ser empregada depende, dentre outros fatores, do tipo de interruptor utilizado no conversor. Para o caso de semicondutores do tipo MOSFET (Metal-Oxide-Semiconductor Field-EffectTransistor) recomenda-se a utilização das técnicas de comutação com tensão nula, em função das capacitâncias intrínsecas de tais dispositivos. Entretanto, o processamento de potência em níveis elevados pode acarretar perdas significativas durante o processo de condução de corrente através dos dispositivos semicondutores.

Assim sendo, por apresentarem menores perdas em condução, interruptores do tipo IGBT (Insulated-Gate Bipolar Transistor) tornam-se mais atrativos que interruptores do tipo MOSFET para o processamento de potência acima de $1 \mathrm{~kW}$ e/ou tensões acima de 500V [10-12]. Contudo, os IGBTs têm como característica a existência de "corrente de cauda" durante o processo de bloqueio, fazendo com que as perdas de comutação tornem-se pronunciadas.
Para contornar tal problema, recomenda-se o emprego das técnicas de comutação com corrente nula, possibilitando então a operação em elevadas freqüências.

Dentro deste contexto, uma célula de comutação ZCSPWM (Pulse Width Modulated) foi proposta em [11], sendo especificamente apresentada em [12] para uma aplicação em um retificador pré-regulador Boost de elevado fator de potência para sistemas de telecomunicações, comandado pela técnica de controle de valores médios instantâneos de corrente de entrada. Diversas propostas de célula ZCS-PWM aplicadas a retificadores pré-reguladores Boost surgiram na literatura, destacando-se as células analisadas em [13] e [14]. A célula apresentada em [13] é aplicada a um conversor Boost interleaved operando no modo de condução crítica de corrente, com o intuito de se minimizar os efeitos de recuperação reversa do diodo Boost sobre o interruptor principal. Já a célula apresentada em [14] possui um indutor ressonante em série com o caminho da corrente transferida à carga, implicando em perdas adicionais neste elemento. Portanto, para a aplicação de técnicas de controle no modo de condução contínua, com minimização dos efeitos de recuperação reversa do diodo Boost sobre os interruptores ativos, a célula apresentada em [11] ainda representa uma escolha adequada. Neste contexto, desde a proposição da célula original [11], três diferentes versões foram seqüencialmente propostas [15 e 16, 17 e 18, 19], cada uma delas mantendo as principais características de comutação suave nos elementos semicondutores empregados e trazendo melhorias adicionais a cada modificação, com a finalidade de elevar o rendimento da estrutura e permitir a redução dos esforços nos semicondutores. Desta forma, este artigo apresenta a seqüência de modificações incorporadas à célula ZCS-PWM original, provendo subsídios para uma comparação aprimorada entre as principais características das diferentes versões propostas, aplicadas a um retificador préregulador Boost de elevado fator de potência para sistemas de telecomunicações.

\section{CÉLULA ORIGINAL DE COMUTAÇÃO ZCS-PWM}

A figura 3 mostra a célula original de comutação ZCSPWM [12], aplicada a um retificador Boost. Com base nesta figura, é possível notar que a célula de comutação proposta apresenta dois interruptores ativos bidirecionais em corrente $\left(S_{1}\right.$ e $\left.S_{2}\right)$, dois diodos $\left(D_{1}\right.$ e $\left.D_{2}\right)$, dois pequenos indutores ressonantes $\left(\mathrm{L}_{\mathrm{r} 1}\right.$ e $\left.\mathrm{L}_{\mathrm{r} 2}\right)$ e um capacitor ressonante $\left(\mathrm{C}_{\mathrm{r}}\right)$.

As etapas de funcionamento para o circuito simplificado e as principais formas de onda teóricas associadas a este estágio retificador são mostradas na figura 4.

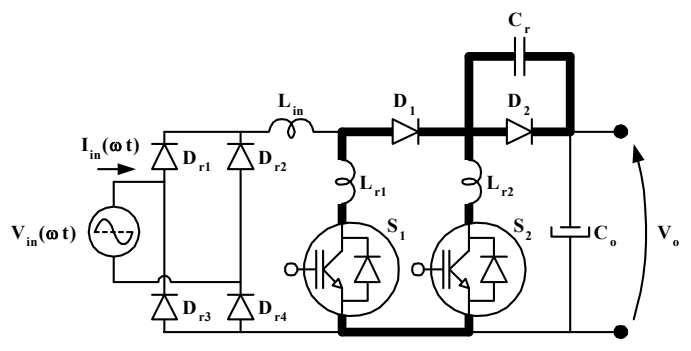

Figura 3 - Estágio retificador Boost, empregando a célula de comutação ZCS-PWM original [12]. 


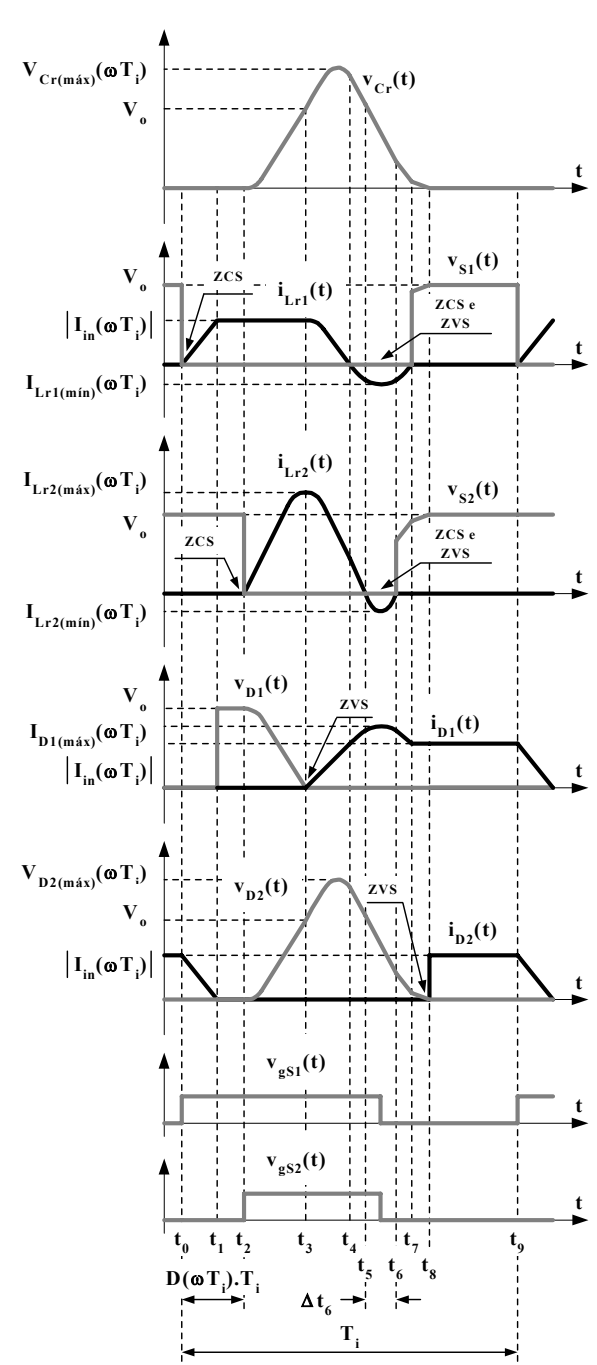

(a)
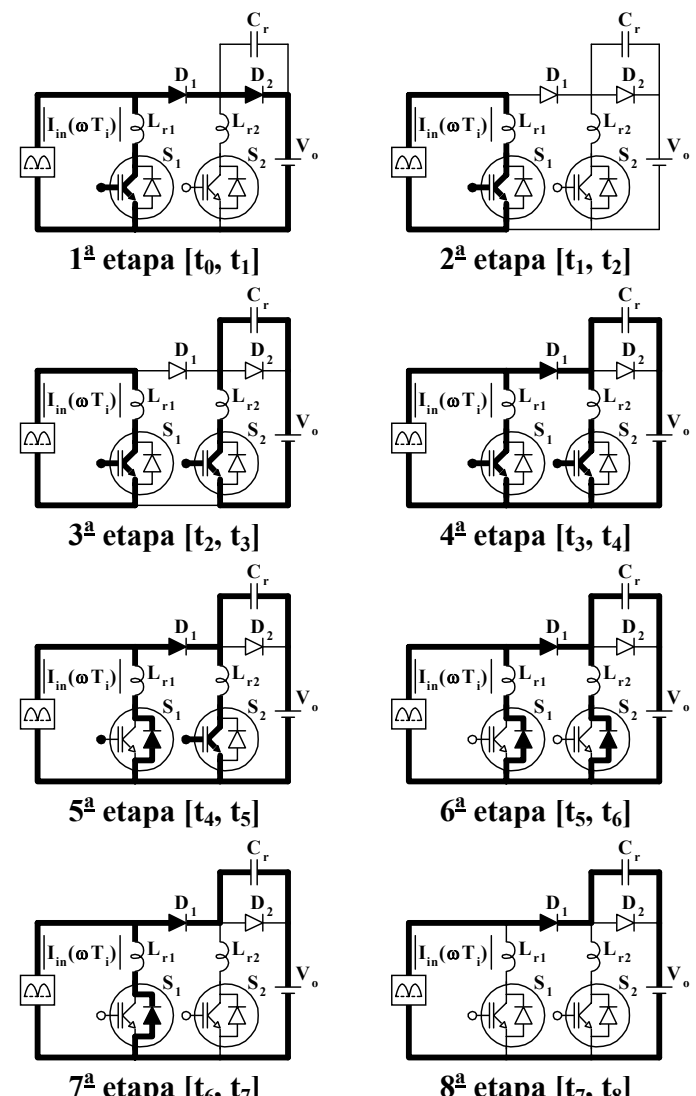

$\mathbf{8}^{\mathrm{a}}$ etapa $\left[\mathbf{t}_{7}, \mathbf{t}_{8}\right]$

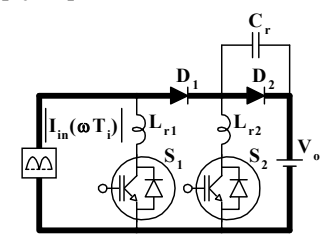

$9^{2}$ etapa $\left[t_{8}, t_{9}\right]$

(b)

Figura 4 - (a) Principais formas de ondas idealizadas; e (b) Etapas de funcionamento do retificador Boost ZCS-PWM com elevado fator de potência [12], durante um período genérico de chaveamento $\left(\mathrm{T}_{\mathrm{i}}\right)$.

De acordo com a figura 4, os interruptores $S_{1}$ e $S_{2}$ são comandados à condução de forma $\mathrm{ZCS}$, em $\mathrm{t}=\mathrm{t}_{0}$ e $\mathrm{t}=\mathrm{t}_{3}$, respectivamente. Tal fato ocorre devido à presença dos indutores $\mathrm{L}_{\mathrm{r} 1}$ e $\mathrm{L}_{\mathrm{r} 2}$, os quais limitam as derivadas das correntes em seus respectivos ramos. Adicionalmente, $\mathrm{S}_{1}$ e $\mathrm{S}_{2}$ são bloqueados, simultaneamente, durante o intervalo de tempo $\Delta \mathrm{t}_{6}$, de forma ZCS e ZVS.Com relação aos diodos $\mathrm{D}_{1}$ e $\mathrm{D}_{2}$, nota-se que seus processos de entrada em condução ocorrem de forma ZVS e os efeitos de recuperação reversa sobre os interruptores ativos são minimizados.

Resultados experimentais apresentados em [12] mostram que a correção do fator de potência da estrutura pode ser realizada através da técnica de controle por valores médios instantâneos de corrente de entrada, mantendo-se as características das comutações suaves nos dispositivos semicondutores, durante todo um período da rede de alimentação em CA.

Apesar do bom desempenho verificado nesta topologia, a célula de comutação proposta apresenta duas características que podem ser apontadas como desvantagens. A primeira delas relaciona-se com o emprego da célula em outros conversores. Conforme [15], a aplicação da célula ZCSPWM original nos conversores Buck-Boost, Sepic e Zeta impossibilita a obtenção de isolamento "natural" através dos indutores de acumulação destas estruturas. A segunda desvantagem é verificada no próprio conversor Boost, onde é possível notar que os diodos $\mathrm{D}_{1}$ e $\mathrm{D}_{2}$ conduzem simultaneamente a corrente que flui da fonte de alimentação para a carga, durante a primeira e a nona etapas. Desta forma as perdas em condução associadas a estes componentes podem se tornar significativas, dependendo do valor da corrente processada através dos mesmos.

\section{PRIMEIRA VARIAÇÃO TOPOLÓGICA DA CÉLULA DE COMUTAÇÃO ZCS-PWM}

Em conformidade com [15], com o intuito de se eliminar a desvantagem referente à obtenção de isolamento "natural" das estruturas Buck-Boost, Sepic e Zeta, propõe-se então uma alteração na célula de comutação ZCS-PWM original, resultando na topologia apresentada na figura 5. Nesta estrutura, quando comparada à célula original, a seqüência das etapas de funcionamento não sofre alterações. No entanto, a ressonância ocorre sem que haja a necessidade de fluxo da corrente ressonante através do capacitor de filtro da tensão de saída, como mostra a figura 6. 


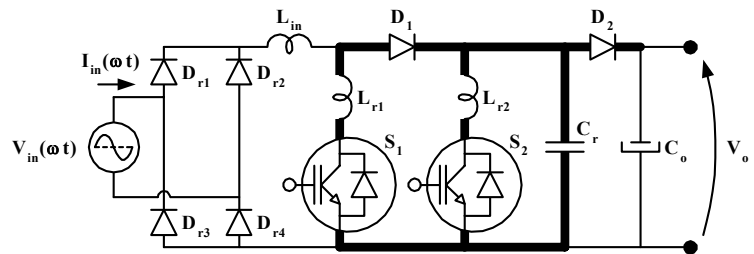

Figura 5 - Estágio retificador Boost, empregando a primeira variação da célula ZCS-PWM [15].

As formas de ondas desta versão do conversor são idênticas àquelas apresentadas para a célula original, à exceção da tensão sobre $C_{r}$, que passa a evoluir conforme a figura 7. Nota-se na figura 7 que a máxima tensão sobre o capacitor ressonante é menor do que aquela verificada para o mesmo elemento na célula original, apesar do valor pico-apico ter permanecido inalterado. Desta forma, pode-se afirmar que a variação proposta para a célula original pode propiciar a redução de custos associados ao elemento $\mathrm{C}_{\mathrm{r}}$, em função do menor nível de isolação de tensão requerido. A exemplo da célula ZCS-PWM original, resultados experimentais para um protótipo implementado confirmam a correção do fator de potência e obtenção de elevado rendimento a partir da estrutura proposta, conforme [15-16]. Entretanto, em comparação com a célula ZCS-PWM original, a conexão série entre os diodos $D_{1}$ e $D_{2}$ durante a etapa de transferência de energia à carga é mantida, sendo esta a sua grande desvantagem. Entretanto, em [16] é apresentada a aplicação desta mesma célula em um retificador Zeta, a partir do qual é possível verificar que os diodos $\mathrm{D}_{1}$ e $\mathrm{D}_{2}$ não conduzem de forma simultânea a corrente de carga.

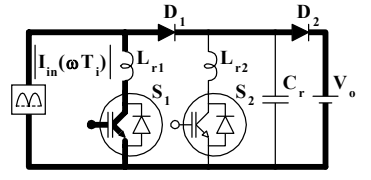

$1^{\mathrm{a}}$ etapa $\left[\mathrm{t}_{0}, \mathrm{t}_{1}\right]$

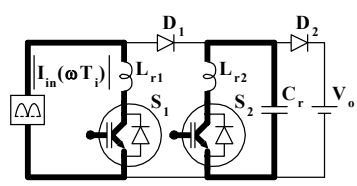

$3^{\mathbf{a}}$ etapa $\left[\mathbf{t}_{2}, \mathbf{t}_{3}\right]$

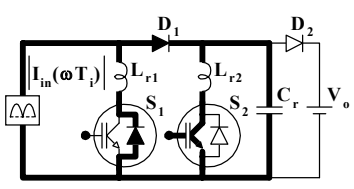

$5^{\mathrm{a}}$ etapa $\left[\mathrm{t}_{4}, \mathrm{t}_{5}\right]$

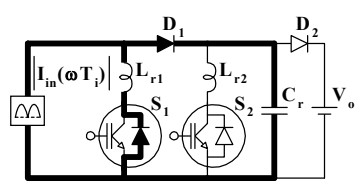

$7^{\mathrm{a}}$ etapa $\left[\mathrm{t}_{6}, \mathrm{t}_{7}\right]$

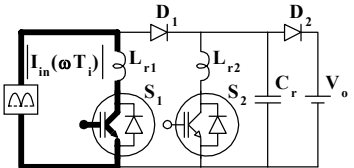

$2^{-\mathbf{a}}$ etapa $\left[\mathbf{t}_{1}, \mathbf{t}_{2}\right]$

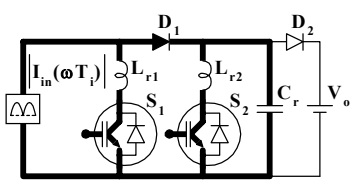

$4^{\mathrm{a}}$ etapa $\left[\mathbf{t}_{3}, \mathrm{t}_{4}\right]$

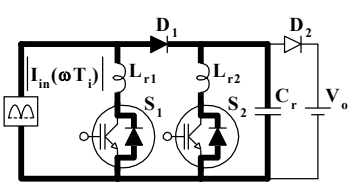

$6^{-}$etapa $\left[t_{5}, t_{6}\right]$

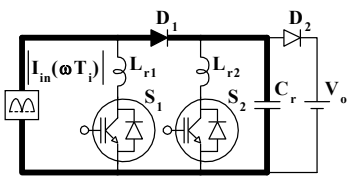

$8^{\text {a }}$ etapa $\left[t_{7}, t_{8}\right]$

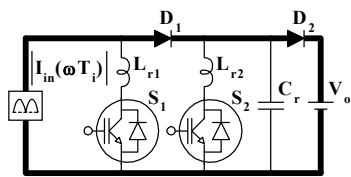

9- etapa $\left[t_{8}, t_{9}\right]$

Figura 6 - Etapas de funcionamento do retificador Boost empregando a primeira variação da célula ZCS-PWM [15], durante um período genérico de chaveamento $\left(T_{\mathfrak{i}}\right)$.

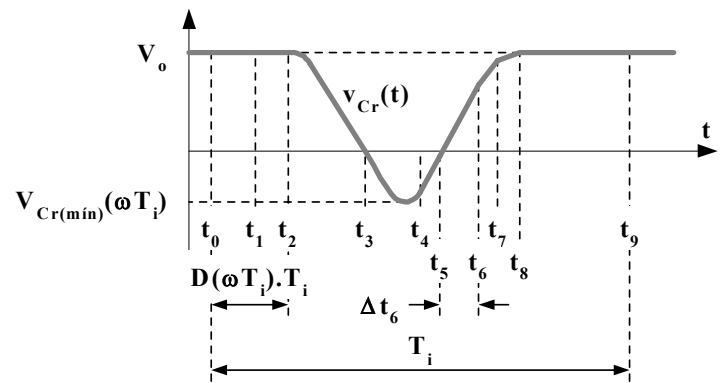

Figura 7 - Forma de onda idealizada da tensão sobre o capacitor ressonante da primeira variação da célula ZCS-PWM

[15], durante um período genérico de chaveamento $\left(T_{i}\right)$.

\section{SEGUNDA VARIAÇÃO TOPOLÓGICA DA CÉLULA DE COMUTAÇÃO ZCS-PWM}

Em função da aplicação explorada em [16], uma segunda variação topológica para a célula original é proposta em [17]. Esta célula é empregada em um estágio retificador isolado Sepic ZCS-PWM de elevado fator de potência, aplicado a reatores eletrônicos para múltiplas lâmpadas fluorescentes [17], e em sua versão não isolada em [18]. Entretanto, tal célula de comutação exige a implementação de circuitos de acionamento (ataque de "gate") isolados, implicando em maior complexidade e custo para o comando. Para solucionar tal problema, em [19] é proposta uma modificação na configuração da célula, na qual os interruptores ativos do estágio retificador apresentam referência comum. É fato que a célula apresentada em [19] pode ser diretamente aplicada para a implementação de um retificador Boost ZCS-PWM, tendo em vista as similaridades de funcionamento com o conversor Sepic ZCS-PWM proposto. A figura 8 apresenta o diagrama esquemático do novo retificador proposto.

As etapas de funcionamento do retificador Boost ZCSPWM mostrado na figura 8 são apresentadas na figura 9.

A figura 10 mostra as formas de onda idealizadas das correntes e tensões nos diodos $\mathrm{D}_{1}$ e $\mathrm{D}_{2}$. As formas de ondas restantes permanecem idênticas àquelas verificadas na primeira variação topológica da célula ZCS-PWM.

Comparando-se as etapas de funcionamento da figura 9 com as etapas do conversor Boost predecessor, mostradas na figura 6, é possível observar que existem diferenças apenas entre as etapas 1 e 9. Entretanto, vale lembrar que, em ambos os conversores, a transferência de energia à carga ocorre durante estas duas etapas, sendo que a etapa 9 é responsável pela maior parte desta transferência. Assim sendo, é fato que a eliminação da conexão série entre os diodos $D_{1}$ e $D_{2}$ na versão da célula mostrada na figura 8 representa então uma grande vantagem com relação à minimização das perdas em condução associadas a $\mathrm{D}_{1}$.

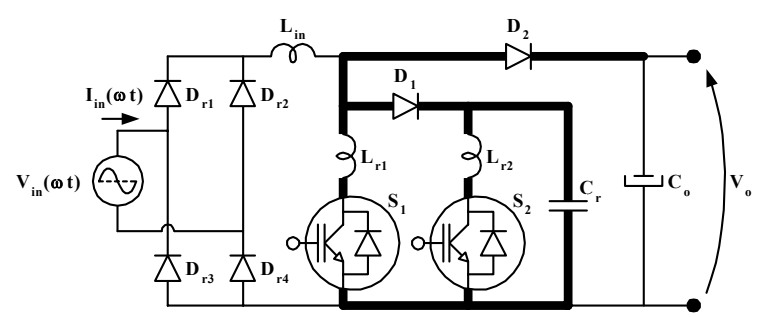

Figura 8 - Estágio retificador Boost, empregando a segunda variação da célula ZCS-PWM [19]. 


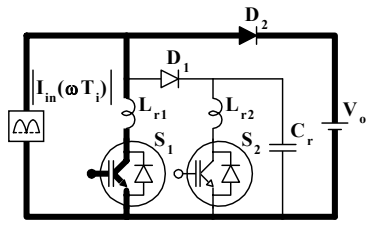

$1^{-}$etapa $\left[\mathbf{t}_{0}, \mathbf{t}_{1}\right]$

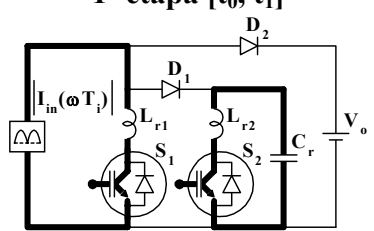

$3^{\mathrm{a}}$ etapa $\left[\mathbf{t}_{2}, \mathbf{t}_{3}\right]$

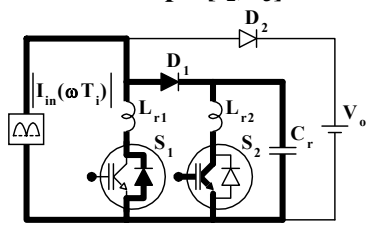

$5^{\mathrm{a}}$ etapa $\left[\mathbf{t}_{4}, \mathbf{t}_{5}\right]$

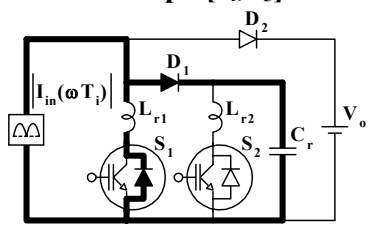

$7^{\mathrm{a}}$ etapa $\left[\mathbf{t}_{6}, \mathbf{t}_{7}\right]$

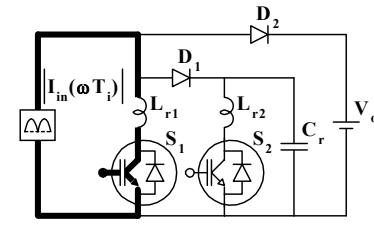

$2^{\mathrm{a}}$ etapa $\left[\mathrm{t}_{1}, \mathrm{t}_{2}\right]$

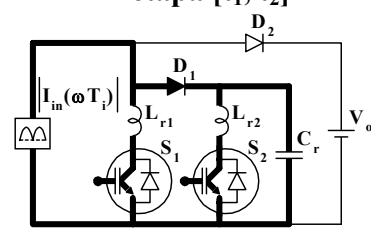

$4^{\mathrm{a}}$ etapa $\left[t_{3}, t_{4}\right]$

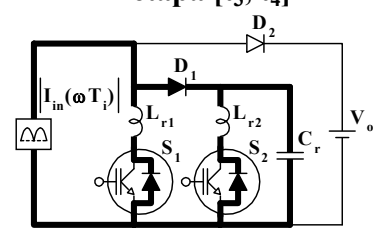

$6^{-}$etapa $\left[t_{5}, t_{6}\right]$

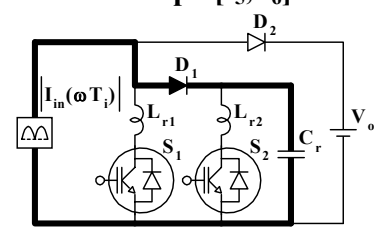

$8^{\mathbf{a}}$ etapa $\left[\mathbf{t}_{7}, \mathbf{t}_{8}\right]$

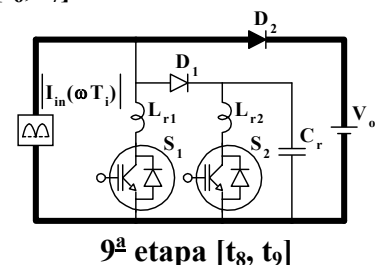

Figura 9 - Etapas de funcionamento do retificador Boost empregando a segunda variação da célula ZCS-PWM [19],

durante um período genérico de chaveamento $\left(T_{i}\right)$.

A Tabela I mostra, de forma resumida, uma comparação entre as principais características das células de comutação ZCS-PWM analisadas. De acordo com esta tabela, é fácil notar a evolução na configuração da célula proposta, possibilitando o isolamento "natural" dos conversores BuckBoost, Sepic e Zeta, além da eliminação da conexão série dos diodos $\mathrm{D}_{1}$ e $\mathrm{D}_{2}$.

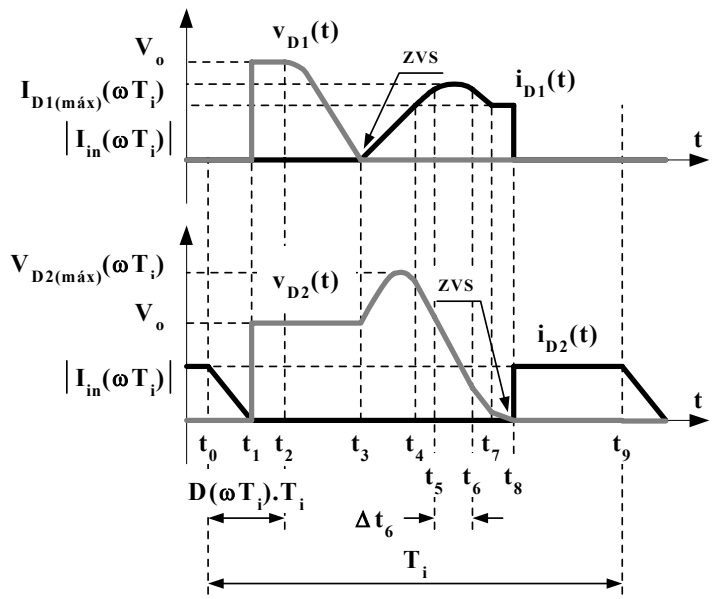

Figura 10 - Formas de ondas idealizadas das comutações dos diodos $D_{1}$ e $D_{2}$ da segunda variação da célula ZCS-PWM [19], durante um período genérico de chaveamento $\left(T_{i}\right)$.
TABELA I

Principais Características das Células ZCS-PWM Analisadas

\begin{tabular}{|c|c|c|c|}
\hline Tipo de Célula & $\begin{array}{c}\text { Fluxo da } \\
\text { Corrente } \\
\text { Ressonante } \\
\text { Através da } \\
\text { Carga }\end{array}$ & $\begin{array}{c}\text { Possibilidade } \\
\text { de Isolamento } \\
\text { das Estruturas } \\
\text { Buck-Boost, } \\
\text { Sepic e Zeta }\end{array}$ & $\begin{array}{c}\text { Conexão } \\
\text { Série dos } \\
\text { Diodos }\end{array}$ \\
\hline Característica & Sim & São \\
\hline
\end{tabular}

\section{EXEMPLO DE PROJETO E RESULTADOS EXPERIMENTAIS}

Para demonstrar a validade da análise desenvolvida, um protótipo do retificador Boost apresentado na figura 8 é implementado, sendo que o circuito de comando para correção do fator de potência é baseado no controlador UC3854 [5], dedicado à técnica por valores médios instantâneos de corrente de entrada.

As principais equações de projeto deste retificador Boost são descritas a seguir. Com o intuito de se garantir a obtenção de comutação ZCS para os interruptores ativos $\mathrm{S}_{1} \mathrm{e}$ $\mathrm{S}_{2}$, conforme descrito anteriormente, é necessário que as restrições impostas pelas inequações (1) e (2) sejam conjuntamente satisfeitas.

$$
\begin{gathered}
\beta=\frac{\mathrm{L}_{\mathrm{r} 2}}{\mathrm{~L}_{\mathrm{r} 1}}<1 \\
\alpha_{\text {máx }}=\frac{\mathrm{I}_{\mathrm{in}(\mathrm{p})}}{\mathrm{V}_{\mathrm{o}}} \cdot \sqrt{\frac{\mathrm{L}_{\mathrm{r} 2}}{\mathrm{C}_{\mathrm{r}}}}<\beta
\end{gathered}
$$

sendo: $\quad I_{\text {in(p) }}=$ valor de pico da corrente de entrada.

$\mathrm{O}$ atendimento das restrições (1) e (2) torna possível o emprego da técnica de controle por valores médios instantâneos de corrente de entrada mantendo-se as comutações suaves da célula ZCS-PWM durante o decorrer de todo um período de rede de CA.

A característica de saída do conversor Boost ZCS-PWM é obtida através de (3).

$$
\begin{gathered}
\mathrm{q}=\frac{\mathrm{V}_{\mathrm{o}}}{\mathrm{V}_{\text {in(ef) }}}=\frac{1}{1-\mathrm{F}} \\
\text { sendo: } \mathrm{F}=\mathrm{D}_{(\mathrm{ef})}+\frac{\mathrm{f}}{2 \cdot \pi} \cdot\left[\mathrm{A}_{0}-\frac{1}{\alpha_{(\mathrm{ef})}} \cdot\left(\mathrm{A}_{1}+\mathrm{A}_{2}\right)+\mathrm{A}_{3}-\frac{\alpha_{(\mathrm{ef})}}{2 \cdot \beta}\right] \\
\mathrm{D}_{(\mathrm{ef})}=\begin{array}{c}
\text { razão cíclica de controle para valores } \\
\text { eficazes de tensão de alimentação }
\end{array}
\end{gathered}
$$




$$
\begin{gathered}
\mathrm{f}=\frac{2 \cdot \pi \cdot \mathrm{f}_{\mathrm{B}}}{\omega_{2}}=2 \cdot \pi \cdot \mathrm{f}_{\mathrm{B}} \cdot \sqrt{\mathrm{L}_{\mathrm{r} 2} \cdot \mathrm{C}_{\mathrm{r}}} \\
\mathrm{A}_{0}=\frac{1}{\alpha_{(\mathrm{ef})}}+\frac{\pi}{2}+\frac{2 \cdot \pi}{\sqrt{1+\beta}} \\
\mathrm{A}_{1}=\sqrt{\beta} \cdot\left(\sqrt{\beta-\alpha_{(\mathrm{ef})}^{2}}-\alpha \frac{(\mathrm{ef})}{\sqrt{\beta}}\right) \\
\mathrm{A}_{3}=\frac{\sqrt{\beta}}{\beta} \cdot \operatorname{arcsen}\left(\frac{\sqrt{1-\beta}}{\beta} \cdot \sqrt{1-\frac{\left(\mathrm{A}_{1}\right)^{2}}{\beta}}\right)-\frac{\sqrt{1+\beta}}{1+\beta} \cdot \arccos (-\beta)
\end{gathered}
$$

Recomenda-se que valores para $\beta$ e f sejam adotados de tal forma que proporcionem a obtenção de reduzida influência da ressonância sobre a regulação da tensão de saída, além de evitar a ocorrência dos problemas relacionados a elevadas freqüências de ressonância, resultando em perdas mais acentuadas nos elementos magnéticos e problemas de interferência eletromagnética.

\section{A. Exemplo de Projeto do Novo Retificador Boost ZCS- PWM com Elevado Fator de Potencia}

O projeto do novo retificador Boost ZCS-PWM é desenvolvido a partir dos dados de entrada e saída definidos na Tabela II

A obtenção dos valores dos elementos ressonantes é realizada com base na adoção dos seguintes parâmetros:

$$
\beta=0,625 ; \mathrm{f}=0,147 \text { e } \alpha_{\text {máx }}=0,51 \text {. }
$$

Utilizando-se os valores acima adotados e com o uso das equações (1) a (9), são então determinados:

$$
\mathrm{C}_{\mathrm{r}}=22 \mathrm{nF} ; \mathrm{L}_{\mathrm{r} 1}=16 \mu \mathrm{H} \text { e } \mathrm{L}_{\mathrm{r} 2}=10 \mu \mathrm{H} \text {. }
$$

$\mathrm{O}$ filtro de entrada $\left(\mathrm{L}_{\text {in }}\right)$ é projetado para que o ripple da corrente de entrada fique limitado a $10 \%$ de seu valor nominal de pico. Quanto ao filtro de saída $\left(\mathrm{C}_{\mathrm{o}}\right)$, seu valor é determinado para que a tensão de saída do conversor apresente um ripple inferior a $2 \%$ de seu valor médio nominal. Assim sendo, são especificados:

$$
\mathrm{L}_{\text {in }}=3 \mathrm{mH} \text { e } \mathrm{C}_{\mathrm{o}}=680 \mu \mathrm{F} .
$$

O projeto dos parâmetros externos do controlador UC3854 é realizado de acordo com [5]. A lógica de acionamento dos interruptores $S_{1}$ e $S_{2}$ é composta a partir do diagrama de blocos da figura 11.

\section{B. Resultados Experimentais}

Resultados experimentais obtidos para um protótipo do retificador Boost da figura 8 são apresentados na seqüência. Para a implementação do protótipo, os dispositivos semicondutores foram especificados conforme a Tabela III.

As formas de ondas da corrente de entrada e da tensão de alimentação do retificador, para condições nominais de operação, são mostradas na figura 12.a. A figura 12.b mostra

$$
\text { TABELA II }
$$

Dados de Entrada e Saída do Retificador Boost ZCS-PWM

\begin{tabular}{|c|c|}
\hline Tensão eficaz de alimentação $\left(\mathrm{V}_{\text {in }(\mathrm{e})}\right)$ & $220 \mathrm{~V} \pm 15 \%$ \\
\hline Freqüência de oscilação da rede de alimentação em CA $\left(\mathrm{f}_{\mathrm{CA}}\right)$ & $60 \mathrm{~Hz}$ \\
\hline Freqüência de chaveamento do retificador Boost $\left(\mathrm{f}_{\mathrm{B}}\right)$ & $50 \mathrm{kHz}$ \\
\hline Valor médio da tensão de saída CC do retificador $\left(\mathrm{V}_{\mathrm{o}}\right)$ & $400 \mathrm{~V}$ \\
\hline Potência nominal de saída $\left(\mathrm{P}_{\mathrm{o}}\right)$ & $1200 \mathrm{~W}$ \\
\hline Mínimo rendimento adotado $(\eta \%)$ & $95 \%$ \\
\hline
\end{tabular}

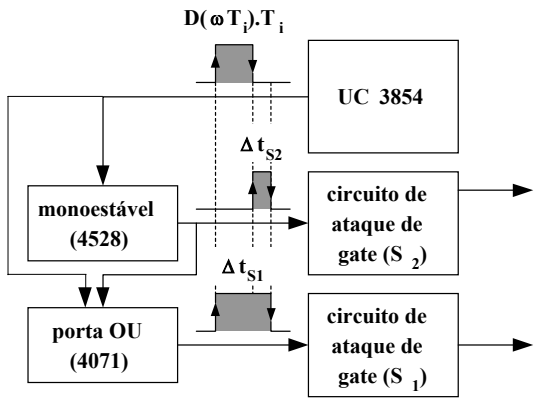

Figura 11 - Diagrama de blocos da lógica de acionamento dos interruptores $S_{1}$ e $S_{2}$.

TABELA III

Dispositivos Semicondutores empregados no Protótipo
\begin{tabular}{|c|c|c|}
\hline \multirow{2}{*}{ Ponte Retificadora } & $\mathrm{D}_{\mathrm{r} 1}, \mathrm{D}_{\mathrm{r} 2}$ & SKR $25 / 06$ \\
\cline { 2 - 3 } & $\mathrm{D}_{\mathrm{r} 3}, \mathrm{D}_{\mathrm{r} 4}$ & SKN $25 / 06$ \\
\hline Interruptor Principal & $\mathrm{S}_{1}$ & IRG4PH50UD \\
\hline Interruptor Auxiliar & $\mathrm{S}_{2}$ & IRG4PC $50 \mathrm{UD}$ \\
\hline Diodo de Equalização & $\mathrm{D}_{1}$ & MUR $8100 \mathrm{E}$ \\
\hline Diodo Boost & $\mathrm{D}_{2}$ & MUR $8100 \mathrm{E}$ \\
\hline
\end{tabular}

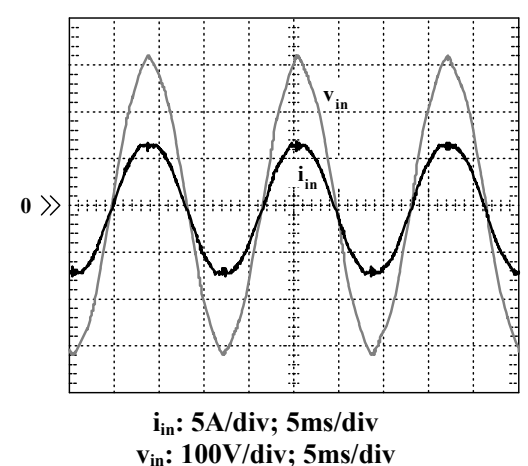

(a)

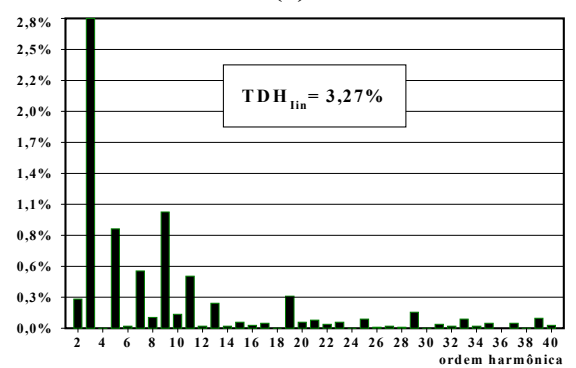

(b)

Figura 12 - (a) Tensão de alimentação e corrente de entrada, e (b) espectro de freqüências de $\mathbf{I}_{\mathrm{i}}$, para carga nominal.

o espectro de freqüências para a corrente de entrada, sendo que sua TDH é igual a 3,27\% e o fator de potência da estrutura nesta condição é de aproximadamente 0,986 , para uma TDH da tensão de alimentação medida de $2,10 \%$.

A Figura 13 mostra os detalhes das comutações dos semicondutores empregados no retificador Boost ZCS-PWM.

Os resultados mostrados na figura 13 foram obtidos para as situações em que o valor instantâneo da tensão de alimentação é próximo de zero $\left(\mathrm{V}_{\text {in }}(\omega \mathrm{t}) \cong 0\right)$ e próximo ao valor de pico $\left(\mathrm{V}_{\text {in }}(\omega \mathrm{t}) \cong \mathrm{V}_{\text {in(p) }}\right)$, para a operação nominal.

De acordo com a figura 13, nota-se que os interruptores $S_{1}$ e $S_{2}$ entram em condução de forma ZCS e são bloqueados de forma ZCS e ZVS, sendo que tais comutações são preservadas durante o decorrer de todo um período de rede em CA, conforme esperado. 


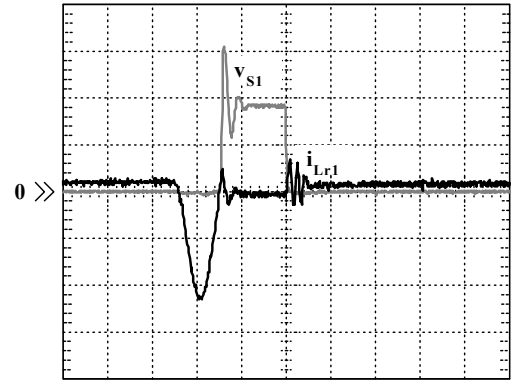

$\mathrm{i}_{\mathrm{Lr} 1}: 5 \mathrm{~A} / \mathrm{div} ; 2 \mu \mathrm{s} / \mathrm{div}$

$\mathrm{v}_{\mathrm{S} 1}: 200 \mathrm{~V} / \mathrm{div} ; 2 \mu \mathrm{s} / \mathrm{div}$

$\mathrm{V}_{\text {in }}(\omega t) \cong 0$

(a) interruptor principal

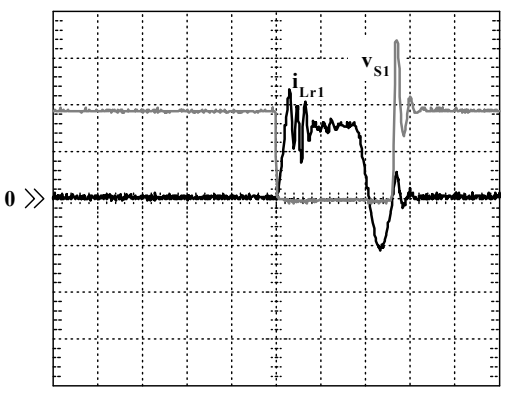

$i_{L r 1}: 5 \mathrm{~A} / \mathrm{div} ; 2 \mu \mathrm{s} / \mathrm{div}$

$\mathrm{v}_{\mathrm{S} 1}: 200 \mathrm{~V} / \mathrm{div} ; 5 \mu \mathrm{s} / \mathrm{div}$

$\mathbf{V}_{\text {in }}(\omega t) \cong V_{\text {in(pk) }}$

(d) interruptor principal

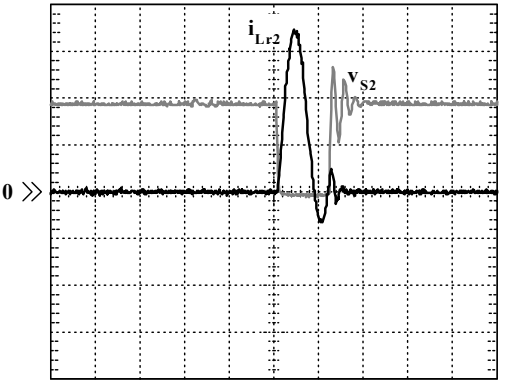

$\mathrm{i}_{\mathrm{Lr} 2}: 5 \mathrm{~A} / \mathrm{div} ; 2 \mu \mathrm{s} / \mathrm{div}$

$\mathrm{v}_{\mathrm{S} 2}: 200 \mathrm{~V} / \mathrm{div} ; 2 \mu \mathrm{s} / \mathrm{div}$

$V_{\text {in }}(\omega t) \cong 0$

(b) interruptor auxiliar

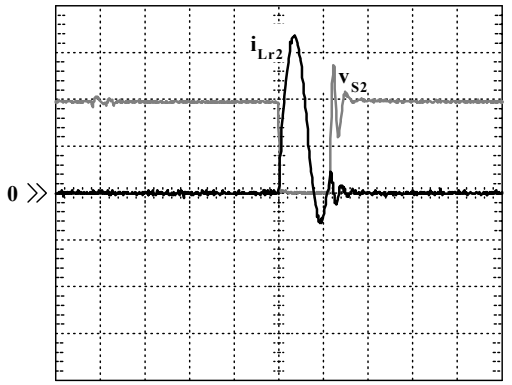

$\mathrm{i}_{\mathrm{Lr} 2}: 5 \mathrm{~A} / \mathrm{div} ; 2 \mu \mathrm{s} / \mathrm{div}$

$\mathrm{v}_{\mathrm{S} 2}: 200 \mathrm{~V} / \mathrm{div} ; 5 \mu \mathrm{s} / \mathrm{div}$

$V_{\text {in }}(\omega t) \cong V_{\text {in(pk) }}$

(e) interruptor auxiliar

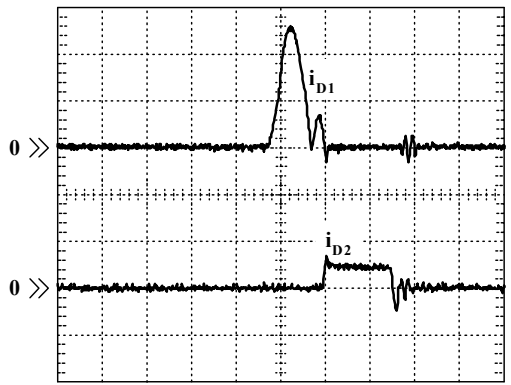

$i_{D 1}: 5 \mathrm{~A} / \mathrm{div} ; 2 \mu \mathrm{s} / \mathrm{div}$

$i_{D 2}: 5 \mathrm{~A} / \mathrm{div} ; 2 \mu \mathrm{s} / \mathrm{div}$

$V_{\text {in }}(\omega t) \cong 0$

(c) diodos $D_{1}$ e $D_{2}$

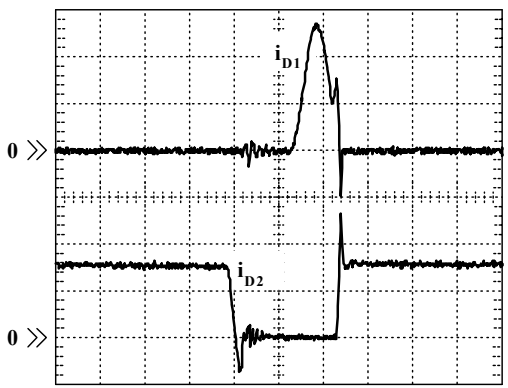

$i_{D 1}: 5 \mathrm{~A} / \mathrm{div} ; 2 \mu \mathrm{s} / \mathrm{div}$

$i_{D 2}: 5 \mathrm{~A} / \mathrm{div} ; 5 \mu \mathrm{s} / \mathrm{div}$

$\mathrm{V}_{\text {in }}(\omega \mathrm{t}) \cong \mathrm{V}_{\text {in(pk) }}$

(f) diodos $\mathrm{D}_{1} \mathrm{e} \mathrm{D}_{2}$

Figura 13 - Formas de ondas de tensão e corrente através dos dispositivos semicondutores, para operação em condições nominais.

Ainda na figura 13, observa-se que os diodos $\mathrm{D}_{1}$ e $\mathrm{D}_{2}$ não apresentam condução simultânea de corrente, caracterizando a redução das perdas em condução associadas a $\mathrm{D}_{1}$, em relação às outras versões da célula de comutação ZCS-PWM.

Em função de todos estes fatos, o rendimento da estrutura deve resultar bastante elevado.

A figura 14 mostra uma comparação entre valores de rendimentos medidos para protótipos de pré-reguladores Boost empregando três diferentes células de comutação, a saber: célula "hard" (Fig. 2), primeira variação da célula ZCS-PWM [15] e versão atual da célula ZCS-PWM [19]. Todos os protótipos foram implementados de acordo com os dados das Tabelas II e III e os intrumentos utilizados para a obtenção das medidas foram voltímetros e amperímetros Yokogawa, classe $0,5 \%$.

Com base nos resultados da figura 14, é possível notar a elevação no rendimento do pré-regulador Boost, em função da utilização da célula ZCS-PWM sem a conexão série entre os diodos $\mathrm{D}_{1}$ e $\mathrm{D}_{2}$. Tendo em vista que a energia destinada à ressonância é constante nas células ZCS-PWM analisadas,

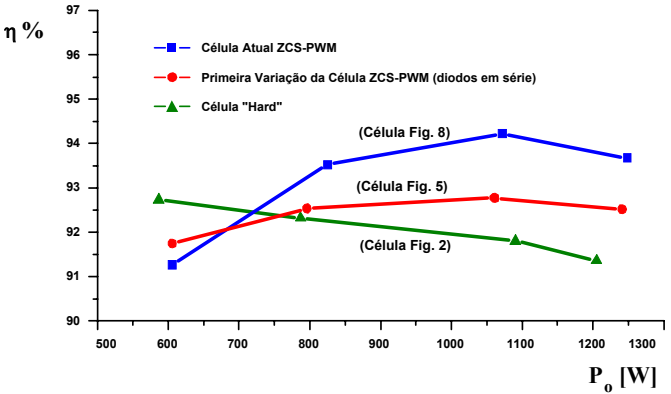

Figura 14 - Comparação entre valores de rendimento medidos. o rendimento dos pré-reguladores que empregam tais células tende a diminuir, conforme a carga conectada à saída também diminui, diferentemente do pré-regulador com célula "hard", onde a redução dos esforços de corrente e a conseqüente redução das perdas em condução levam a um aumento do rendimento, quando do processamento de menores cargas.

\section{CONCLUSÕES}

Este artigo apresentou um resumo sobre a evolução de uma célula de comutação ZCS-PWM aplicada a estágios retificadores Boost de elevado fator de potência.

As principais características de cada uma das células são apresentadas e discutidas, provendo informações suficientes para uma comparação detalhada entre as estruturas propostas.

A célula ZCS-PWM original apresenta como limitação o fato de não permitir o isolamento das estruturas Buck-Boost, Sepic e Zeta através de seus indutores de acumulação, além de possuir uma etapa de funcionamento em que a corrente da fonte de alimentação flui para a carga através de dois diodos conectados em série. A proposição das versões subseqüentes da célula ZCS-PWM possibilitou, gradativamente, a eliminação das desvantagens apontadas na célula original, mantendo-se todas as características de comutações nãodissipativas nos dispositivos semicondutores utilizados.

Um protótipo do retificador Boost empregando a última versão da célula ZCS-PWM foi implementado para a verificação da análise desenvolvida. A partir dos resultados obtidos, conclui-se que a estrutura proposta opera conforme esperado, propiciando comutações suaves em todos os 
dispositivos semicondutores, além da eliminação da conexão série dos diodos $\mathrm{D}_{1}$ e $\mathrm{D}_{2}$.

A técnica de controle por valores médios de corrente de entrada é empregada com sucesso no estágio retificador Boost ZCS-PWM projetado, fornecendo reduzida TDH e defasagem angular desprezível na corrente de entrada, em relação à tensão de alimentação em $\mathrm{CA}$, resultando em fator de potência praticamente unitário.

A respeito do rendimento da estrutura, o resultado obtido para carga nominal, considerando-se os semicondutores empregados e a instrumentação utilizada para análise, mostrou-se elevado.

Por fim, a obtenção de elevado rendimento e fator de potência praticamente unitário denota o excelente aproveitamento da energia drenada da rede de alimentação em CA, em função do emprego da estrutura proposta.

\section{AGRADECIMENTOS}

Os autores agradecem à FAPESP pelo apoio concedido ao desenvolvimento deste trabalho.

\section{REFERÊNCIAS BIBLIOGRÁFICAS}

[1] M. J. Korcher e R. L. Steigerwald, "An AC-to-DC Converter with High Quality Input Waveforms", Anais do IEEE PESC, 1982, pp. 63-75.

[2] M. F. Schlecht e B. A. Miwa, "Active Power Factor Correction for Switching Power Supplies", IEEE Transactions on Power Electronics, Outubro, 1987, pp. 273-281.

[3] K. Sen e A. E. Emanuel, "Unity Power Factor Single Phase Power Conditioning", Anais do IEEE PESC, 1987, pp. 516-524.

[4] R. Erickson, M. Madigan e S. Singer, "Design of a Simple High-Power-Factor Rectifier based on the Flyback Converter", Anais do IEEE APEC, 1990, pp. 792-801.

[5] C. S. E. Silva, "Power Factor Correction with the UC3854", Application Note U125, Unitrode Corporation, 1990, pp 287-296.

[6] C. A. Canesin e I. Barbi, "A Unity Power-FactorMultiple isolated Outputs Switching Mode Power Supply using a Single Switch", Anais do IEEE APEC, 1991, pp. 430-436.

[7] S. A. O. da Silva e I. Barbi, "Sinusoidal Line Current Rectification at Unity Power Factor with Boost Quasi Resonant Converter", Anais do IEEE APEC, 1990, pp. 553-562.

[8] R. Streit e D. Tollik, "High Efficiency Telecom Rectifier using a Novel Soft-Switched Boost-Based Input Current Shaper", Anais do IEEE INTELEC, 1991, pp. 720-726.

[9] G. Hua, C. S. Leu e F. C. Lee, "Novel Zero-VoltageTransition PWM Converters", Anais do IEEE PESC, 1992, pp. 55-61.

[10] K. Wang, G. Hua, e F. C. Lee, "Analysis, Design and Experimental Results of ZCS-PWM Boost Converters", Anais do IEEJ IPEC, 1995, pp. 1197-1202.

[11] C. A. Canesin e I. Barbi, "Comparison of Experimental Losses among Six Different Topologies for a $1.6 \mathrm{~kW}$
Boost Converter, using IGBTs", Anais do IEEE PESC, 1995, pp. 1265-1271.

[12] C. A. Canesin e I. Barbi, "Um Novo Pré-Regulador com Elevado Fator de potência e Comutação ZCSPWM para Fontes de Alimentação de Sistemas de Telecomunicações", Anais do COBEP, Belo Horizonte (MG), 1997, pp. 153-158.

[13] C. M. O. Stein, J. R. Pinheiro, H. L. Hey, "Application of the ZCS-Auxiliary Commutation Circuits in Interleaved Boost Converters operating in Critical Conduction Mode", Anais do IEEE APEC, 2000, CDROM

[14] H. Choi e B. H. Cho, "Zero-Current-Switching (ZCS) Power Factor Pre-regulator (PFP) with Reduced Conduction Losses" Anais do IEEE APEC, 2002, CDROM

[15] F. T. Wakabayashi e C. A. Canesin, "A New Family of Zero-Current-Switching PWM Converters and a Novel HPF-ZCS-PWM Boost Rectifier", Anais do IEEE APEC, 1999, pp. 605-611.

[16] F. T. Wakabayashi, M. J. Bonato e C. A. Canesin, "Novel High-Power-Factor ZCS-PWM Preregulators", IEEE Transactions on Industrial Electronics, vol. 48, $\mathrm{n}^{\mathrm{o}}$ 2, 2001, pp. 322-333.

[17] F. T. Wakabayashi e C. A. Canesin, "Novel HighPower-Factor Isolated Electronic Ballast for Multiple Tubular Fluorescent Lamps", Anais do IEEE IAS Annual Meeting, 2001, CD-ROM.

[18] F. T. Wakabayashi e C. A. Canesin, "Novo Reator Eletrônico com Elevado Fator de Potência para Múltiplas Lâmpadas Fluorescentes Tubulares", Revista Eletrônica de Potência, vol. 6, no 1, 2001, pp. 16-24.

[19] F. T. Wakabayashi e C. A. Canesin, "A High Efficiency HPF-ZCS-PWM Sepic for Electronic Ballast with Multiple Tubular Fluorescent Lamps", Anais do IEEE APEC, 2002, pp. 924- 930.

\section{DADOS BIOGRÁFICOS}

Fabio Toshiaki Wakabayashi, nascido em Jales (SP), em Julho de 1974, é engenheiro eletricista (1996) e mestre em Engenharia Elétrica (1998), formado na Universidade Estadual Paulista - Faculdade de Engenharia de Ilha Solteira (UNESP-FEIS, Ilha Solteira (SP)), onde atualmente desenvolve o doutorado em Eletrônica de Potência. Suas áreas de interesse abrangem técnicas de comutação nãodissipativa, fontes de alimentação chaveadas, qualidade de energia elétrica e reatores eletrônicos para iluminação.

Carlos Alberto Canesin, nascido em Lavínia (SP), em 1961, é engenheiro eletricista (1984) pela Universidade Estadual Paulista - Faculdade de Engenharia de Ilha Solteira (UNESP-FEIS, Ilha Solteira (SP)), mestre (1990) e doutor (1996) em Engenharia Elétrica pela Universidade Federal de Santa Catarina - Instituto de Eletrônica de Potência (UFSCINEP), Florianópolis (SC). Atualmente é professor adjunto efetivo do Departamento de Engenharia Elétrica (DEE) da UNESP-FEIS. Suas áreas de interesse incluem técnicas de comutação não-dissipativa, conversores $\mathrm{CC} / \mathrm{CC}$, fontes de alimentação chaveadas, reatores para iluminação e técnicas de correção do fator de potência. 